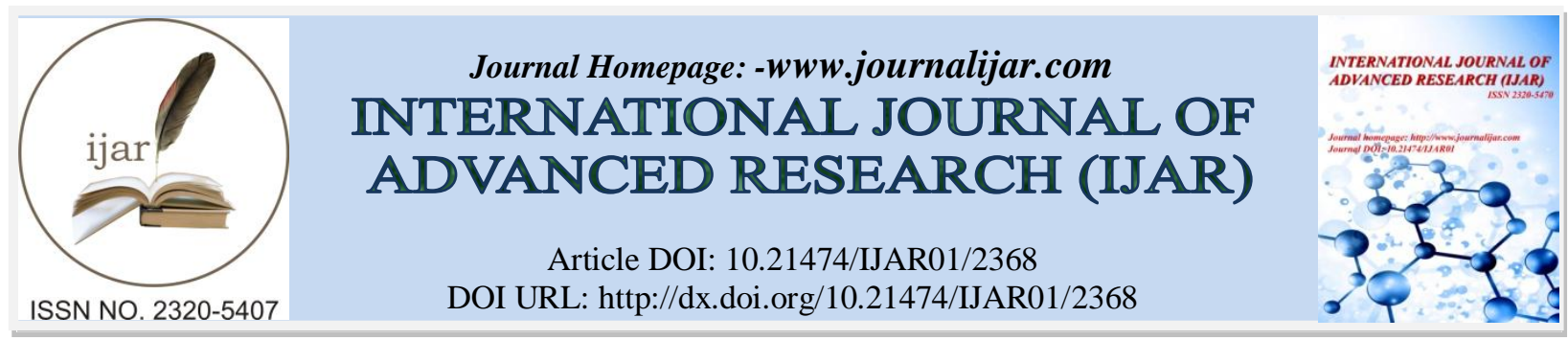

RESEARCH ARTICLE

\title{
PREVALENCE OF FOOT COMPLICATIONSAND FACTORS ASSOCIATED WITH DIABETIC FOOT ULCER IN DIABETIC PATIENTS IN THE QASSIM REGION, SAUDI ARABIA.
}

\section{Fahad Khalid Alshubaily ${ }^{1}$, Hoor Mohammed Al Obaysi ${ }^{2}$, Ali Othman Aldubakhi ${ }^{3}$ and Zayed Ozaywi Al- Resheedi ${ }^{4}$.}

1. Department of Internship Unit, College of Medicine, Qassim University, Kingdom of Saudi Arabia.

2. College of Medicine, Qassim University, Unizah,, Kingdom of Saudi Arabia.

3. Department of Internship Unit, College of Medicine, Qassim University, Kingdom of Saudi Arabia.

4. Diabetic and Endocrinology Center, King Fahad Special Hospital, Qassim region, Kingdom of Saudi Arabia.

\section{Manuscript Info}

Manuscript History

Received: 18 October 2016

Final Accepted: 20 November 2016

Published: December 2016

Key words:-

Diabetes; prevalence; diabetic foot

ulcercomplications, risk factors.

\section{Abstract}

Objectives: The main objective of this study was to assess the prevalence and factors influencing diabetic foot ulcers among diabetic patients, in the Qassim region, Saudi Arabia.

Methodology: A cross-sectional survey was conducted in the Qassim region, Saudi Arabia, between March 2016 and September 2016, involving 817 subjects. A simple random sampling was performed to identify two governmental hospitals in the Qassim region for this study: the King Fahd Specialist Hospital and the King Saud Hospital. All data were recorded and analyzed using SPSS 22, and $\mathrm{p}<0.05$ was considered statistically significant.

Results: The prevalence of diabetic foot ulcers in the Qassim region is $10.8 \%$. There is an increased prevalence among female patients $(12 \%)$ than among male patients $(9.6 \%)$. The prevalence of amputation of a toe, foot, or leg is $2.5 \%(21)$. The prevalence of peripheral neuropathy is $49.45 \%$, and $60 \%$ of patients with peripheral neuropathy are female, while $40 \%$ are male. The following were found to be the risk factors for developing diabetic foot ulcers: duration of DM; use of insulin; lack of school education; sore or cut involving the foot or leg and requiring $>2$ weeks to heal; paresthesia; use of moisturizing cream on the feet; and walking barefoot $(\mathrm{p}<0.05)$.

Conclusion: The prevalence of diabetic foot ulcers in the Qassim Regionis $10.8 \%$, which is within the global prevalence range. The results of this study confirm that DM foot ulcers are caused by multiple factors.

\section{Introduction:-}

Diabetes mellitus (DM) is a chronic and serious disorder affecting a significant number of people worldwide. Many lifestyle-related factors have contributed to the establishment of DM as the disease of the era, including reduced physical activity and poor eating habits, which lead to obesity. Moreover, social factors, including education level, affect diabetes progression. In 2013, almost 382 million people worldwide were affected by DM; this is expected to increase to 592 million by 2035 (1).

Corresponding Author:- Fahad Khalid Alshubaily. 
Eastern Arab countries are among the top 10countries with the highest DM prevalence $(2,3)$. According to recent estimates, 34.6 million people in eastern Arab countries, or 9.2\% of the adult population, have DM. The prevalence ofDM among the younger population in eastern Arab countries is also higher than the global rate. Furthermore, 25.2 million people, or $6.7 \%$ of the population, are estimated to be pre-diabetic and, therefore, at high risk of DM.

It is anticipated that the prevalence of DM will almost double by 2035 (2, 3). In Saudi Arabia, there are 14,900 children with type $1 \mathrm{DM}$; this is the highest prevalence of DM in the region, comprising approximately a quarter of the 64,000children with type $1 \mathrm{DM}$ in the region $(2,3)$. The International Diabetes Federation estimated that $17.6 \%$ (3.4 million) of adults aged 20-79 years had DM in Saudi Arabia in 2015(4). The high prevalence of DM in Saudi Arabia is due to both type 1 and type 2 DM.

In Saudi Arabia, the national health care burden resulting from DM in 2010 was US $\$ 0.9$ billion and this is expected to rise to US $\$ 6.5$ billion by 2020 (5). Accordingly, DM is an important governmental and public issue. Regarding common DM complications, the International Diabetes Federation reports that up to $70 \%$ of all leg amputations occur in patients with DM and85\% of DM-related lower limb amputations are preceded by foot ulcers. It has been estimated that, in developing countries, DM foot complications account for up to $40 \%$ of total available resources (6). The most common complication of DM is diabetic peripheral neuropathy, which requires medical or surgical management (10). Studies in Arab countries have shown a prevalence of neuropathy ranging from 38\% to 94\% among cases of diabetic foot disease (7-9). In Saudi Arabia,65.3\% of adults with DM were found to have diabetic peripheral neuropathy and, of these, $42.3 \%$ were prescribed medication for peripheral neuropathy pain (11).

Hence, diabetic foot complications remain a global issue. In the current study, we assessed the prevalence of diabetic patients undergoing foot care in the Qassim Region, Saudi Arabia; wealsoassessed factors associated with diabetic foot ulcers.

\section{Research Methodology:-}

This was an observational descriptive cross-sectional questionnaire-based study conducted in the Qassim Region, Saudi Arabia. The survey was conducted between March 2016 and September 2016. Ethical approval of the study was obtained from the QassimUniversity Ethical Committee and the research ethics committee for the Qassim Region; written informed consent was obtained from all participants. Permission was obtained to use the Diabetic Foot Care Program of Nova Scotia questionnaire for the current study. The validity and reliability of this questionnaire have been established in previous studies. $(12,13)$. The questionnaire was translated intothe Arabic language and the translation was validated using a two-way translation method. We adjusted the translated questionnaire based on feedback from the diabetic population toimprove the readability of the questions for Saudi diabetic patients. The questionnaire components included the following: 1- socioeconomic status, 2- current foot or leg problems, 3- history of foot problems, 4- physician or physiotherapist follow-up, 5- foot care education, 6- selfmanagement, 7- current foot care, 8- footwear, 9- foot care programs developed by the World Diabetes Foundation, and10- implementation of the programs into daily life.

This study involved two governmental hospitals in the Qassim Region, identified via simple random sampling: the King Fahd Specialist Hospital and the King Saud Hospital. Questionnaires were administered to the patients in the outpatient departmentofthe diabetic and endocrine centers in each hospital. Patients were randomly selected by systemic random sampling (every third patient attending the clinic was selected). Individuals known to bediabetic were included in the study. Patients who could not hear or comprehend instructions or refused to sign the consent form were excluded. The sample size was 817, considering $\alpha$ of $5 \%$, with an expected DM prevalence in the Qassim Region of $10.8 \%$ (47,800 DM patients), obtained from the "Statistics Book of the Ministry of Health for the year $1436 \mathrm{H}$ ", with a margin of error of $\pm 3.4 \%$. All data were recorded and analyzed using SPSS 22 , and $\mathrm{p}<0.05$ was considered statistically significant. In the model developed for this study, the presence of a foot ulcer is the outcome variable, or the dependent variable, while all the other variables are independent variables. The logistic regression model can be expressed as the log of the odds, called the logit model, as:

$$
\log \left\{\frac{\pi}{1-\pi}\right\}=\operatorname{logit}(\pi)=B_{0}+B_{1} X_{1}+B_{2} X_{2}+\ldots+B_{n} X_{n}
$$

where $\pi$ is the probability of an event accruing, which is equal toP(Y), as previously described in the equations, and the odds are given as: 


$$
\frac{\pi}{1-\pi}=\text { odds }=\frac{\operatorname{Pr}(\text { success })}{\operatorname{Pr}(\text { Failure })}=\frac{\operatorname{Pr}(\text { success })}{1-\operatorname{Pr}(\text { success })}
$$

\section{Results:-}

Participant characteristics are reported in Table 1. Of the 817diabetic patients included in the study, 367 (45\%) were female and 450 (55\%) were male. Approximately 16\% were aged <40 years, $22.0 \%$ were aged $40-49$ years, 39.3\% were aged 50-59 years, and $22.5 \%$ were aged $>59$ years. The majority $(94 \%)$ of participants resided in urban areas. Approximately $22.6 \%$ of participants were illiterate, $18.1 \%$ had either a primary school degree or no degree at all, $34.2 \%$ had either a secondary school degree or high school degree, and $25 \%$ had a university degree. Approximately one-third of patients had no income, 57.8\% had an income of SAR 1,00015,000 , and $10 \%$ had an income of $>$ SAR 15,000. The type of DM was unknown to patients in approximately $60 \%$ of cases, while approximately $24 \%$ reported having type 2 DM. Approximately $50.5 \%$ patients had DM duration of 1-10 years, and $23.3 \%$ had DM duration of $>15 y e a r s$. The majority of participants used oral hyperglycemic drugs, while only $25 \%$ of patients used insulin. Only $10.8 \%$ (87) participants reported a positive history of foot ulcers and $2.5 \%$ (21) of diabetic patients had a history of amputation of a toe, foot, or leg.

Table 1:- Demographic variables among DM patients with and without foot ulcer.

\begin{tabular}{|c|c|c|c|c|c|c|}
\hline \multirow[t]{3}{*}{ Variables } & \multirow[t]{3}{*}{ Categories } & \multicolumn{4}{|c|}{ Diabetic patients } & \multirow[t]{3}{*}{$p$-value } \\
\hline & & \multicolumn{2}{|c|}{ Without foot ulcer } & \multicolumn{2}{|c|}{ With foot ulcer } & \\
\hline & & $\mathrm{N}$ & $\%$ & & $\mathrm{~N}$ & \\
\hline \multirow[t]{2}{*}{ Sex } & Female & 323 & $88.0 \%$ & 44 & $12.0 \%$ & \multirow[t]{2}{*}{0.262} \\
\hline & Male & 407 & $90.4 \%$ & 43 & $9.6 \%$ & \\
\hline \multirow[t]{7}{*}{ Age (years) } & $<10$ & 1 & $100.0 \%$ & 0 & $0.0 \%$ & \multirow[t]{7}{*}{0.384} \\
\hline & $10-19$ & 30 & $96.8 \%$ & 1 & $3.2 \%$ & \\
\hline & $20-29$ & 40 & $90.9 \%$ & 4 & $9.1 \%$ & \\
\hline & $30-39$ & 52 & $91.2 \%$ & 5 & $8.8 \%$ & \\
\hline & $40-49$ & 164 & $91.6 \%$ & 15 & $8.4 \%$ & \\
\hline & $50-60$ & 286 & $89.1 \%$ & 35 & $10.9 \%$ & \\
\hline & $>60$ & 157 & $85.3 \%$ & 27 & $14.7 \%$ & \\
\hline \multirow[t]{2}{*}{ Residence } & Rural & 45 & $91.8 \%$ & 4 & $8.2 \%$ & \multirow[t]{2}{*}{0.561} \\
\hline & Urban & 685 & $89.2 \%$ & 83 & $10.8 \%$ & \\
\hline \multirow[t]{6}{*}{ Education status } & Illiterate & 163 & $88.1 \%$ & 22 & $11.9 \%$ & \multirow[t]{6}{*}{0.001} \\
\hline & No formal education & 41 & $74.5 \%$ & 14 & $25.5 \%$ & \\
\hline & Primary degree & 80 & $86.0 \%$ & 13 & $14.0 \%$ & \\
\hline & Secondary degree & 73 & $88.0 \%$ & 10 & $12.0 \%$ & \\
\hline & High school degree & 185 & $93.9 \%$ & 12 & $6.1 \%$ & \\
\hline & University degree & 188 & $92.2 \%$ & 16 & $7.8 \%$ & \\
\hline \multirow[t]{5}{*}{ Monthly income (SAR) } & No salary & 237 & $90.1 \%$ & 26 & $9.9 \%$ & \multirow[t]{5}{*}{0.212} \\
\hline & $1000-5000$ & 127 & $84.1 \%$ & 24 & $15.9 \%$ & \\
\hline & $6000-10,000$ & 167 & $89.8 \%$ & 19 & $10.2 \%$ & \\
\hline & $11,000-15,000$ & 124 & $91.9 \%$ & 11 & $8.1 \%$ & \\
\hline & $>15,000$ & 75 & $91.5 \%$ & 7 & $8.5 \%$ & \\
\hline \multirow[t]{3}{*}{ Type of diabetes } & Type2 & 181 & $92.3 \%$ & 15 & $7.7 \%$ & \multirow[t]{3}{*}{0.088} \\
\hline & Type1 & 118 & $92.2 \%$ & 10 & $7.8 \%$ & \\
\hline & Unknown to patient & 431 & $87.4 \%$ & 62 & $12.6 \%$ & \\
\hline \multirow{6}{*}{ Duration of diabetes (years) } & $<1$ & 69 & $92.0 \%$ & 6 & $8.0 \%$ & \multirow[t]{6}{*}{$<0.001$} \\
\hline & $1-5$ & 206 & $95.4 \%$ & 10 & $4.6 \%$ & \\
\hline & $6-10$ & 180 & $91.4 \%$ & 17 & $8.6 \%$ & \\
\hline & $11-15$ & 125 & $89.9 \%$ & 14 & $10.1 \%$ & \\
\hline & $16-20$ & 70 & $82.4 \%$ & 15 & $17.6 \%$ & \\
\hline & $>20$ & 80 & $76.2 \%$ & 25 & $23.8 \%$ & \\
\hline
\end{tabular}

Among the five clinical manifestations of DM foot complications, the most commonly reported problems were numbness, tingling, pins and needles or itching sensation of the feet (Table2), as described by approximately 
$60 \%$ of female and $40.7 \%$ of male participants. Foot ulcers were more likely to occur among female participants $(39.1 \%)$ than among male (33.3\%) participants. The overall prevalence of numbness, tingling, pins and needles or itching sensation of the feet, known as peripheral neuropathy, was 404 out of 817 (49.45\%). The prevalence of decreased foot sensation to pain and touch was 198 (24.2\%) (Table 3).

Table2. Prevalence of DM foot complications screened during the study.

\begin{tabular}{|c|c|c|c|c|c|c|}
\hline \multirow{3}{*}{\multicolumn{2}{|c|}{ Current foot complication }} & \multicolumn{4}{|c|}{ Sex } & \multirow{3}{*}{$\begin{array}{l}\text { DMD* } \\
\text { duration }\end{array}$} \\
\hline & & \multicolumn{2}{|c|}{ Female } & \multicolumn{2}{|c|}{ Male } & \\
\hline & & $N$ & $\%$ & $N$ & $\%$ & \\
\hline \multirow[t]{5}{*}{$\mathrm{A}$} & Ulcer, sore, or blister on feet & 1 & $1.1 \%$ & 5 & $5.5 \%$ & \multirow[t]{5}{*}{$<1$ year } \\
\hline & Blood or discharge on socks & 0 & $0.0 \%$ & 2 & $6.5 \%$ & \\
\hline & Foot callus & 3 & $4.5 \%$ & 4 & $6.0 \%$ & \\
\hline & $\begin{array}{l}\text { Numbness, tingling, pins and needles or itching } \\
\text { sensation in feet }\end{array}$ & 15 & $3.7 \%$ & 18 & $4.5 \%$ & \\
\hline & Tightness, heaviness, pain, or cramps in feet or legs & 8 & $2.7 \%$ & 11 & $3.7 \%$ & \\
\hline \multirow[t]{5}{*}{$\mathrm{B}$} & Ulcer, sore, or blister on feet & 7 & $7.7 \%$ & 11 & $12.1 \%$ & \multirow[t]{5}{*}{$1-5$ years } \\
\hline & Blood or discharge on socks & 0 & $0.0 \%$ & 4 & $12.9 \%$ & \\
\hline & Foot callus & 6 & $9.0 \%$ & 9 & $13.4 \%$ & \\
\hline & $\begin{array}{l}\text { Numbness, tingling, pins and needles or itching } \\
\text { sensation in feet }\end{array}$ & 39 & $9.7 \%$ & 42 & $10.4 \%$ & \\
\hline & Tightness, heaviness, pain, or cramps in feet or legs & 31 & $10.4 \%$ & 24 & $8.1 \%$ & \\
\hline \multirow[t]{5}{*}{$\mathrm{C}$} & Ulcer, sore, or blister on feet & 8 & $8.8 \%$ & 7 & $7.7 \%$ & \multirow[t]{5}{*}{$6-10$ years } \\
\hline & Blood or discharge on socks & 4 & $12.9 \%$ & 3 & $9.7 \%$ & \\
\hline & Foot callus & 11 & $16.4 \%$ & 10 & $14.9 \%$ & \\
\hline & $\begin{array}{l}\text { Numbness, tingling, pins and needles or itching } \\
\text { sensation in feet }\end{array}$ & 42 & $10.4 \%$ & 41 & $10.1 \%$ & \\
\hline & Tightness, heaviness, pain, or cramps in feet or legs & 30 & $10.1 \%$ & 36 & $12.1 \%$ & \\
\hline \multirow[t]{5}{*}{$\mathrm{D}$} & Ulcer, sore, or blister on feet & 11 & $12.1 \%$ & 8 & $8.8 \%$ & \multirow[t]{5}{*}{$11-15$ years } \\
\hline & Blood or discharge on socks & 3 & $9.7 \%$ & 1 & $3.2 \%$ & \\
\hline & Foot callus & 8 & $11.9 \%$ & 2 & $3.0 \%$ & \\
\hline & $\begin{array}{l}\text { Numbness, tingling, pins and needles or itching } \\
\text { sensation in feet }\end{array}$ & 47 & $11.6 \%$ & 34 & $8.4 \%$ & \\
\hline & Tightness, heaviness, pain, or cramps in feet or legs & 31 & $10.4 \%$ & 26 & $8.7 \%$ & \\
\hline \multirow[t]{5}{*}{$\mathrm{E}$} & Ulcer, sore, or blister on feet & 7 & $7.7 \%$ & 5 & $5.5 \%$ & \multirow[t]{5}{*}{ 16-20 years } \\
\hline & Blood or discharge on socks & 4 & $12.9 \%$ & 2 & $6.5 \%$ & \\
\hline & Foot callus & 3 & $4.5 \%$ & 2 & $3.0 \%$ & \\
\hline & $\begin{array}{l}\text { Numbness, tingling, pins and needles or itching } \\
\text { sensation in feet }\end{array}$ & 38 & $9.4 \%$ & 19 & $4.7 \%$ & \\
\hline & Tightness, heaviness, pain, or cramps in feet or legs & 33 & $11.1 \%$ & 16 & $5.4 \%$ & \\
\hline \multirow[t]{5}{*}{$\mathrm{F}$} & Ulcer, sore, or blister on feet & 8 & $8.8 \%$ & 13 & $14.3 \%$ & \multirow[t]{5}{*}{$>20$ years } \\
\hline & Blood or discharge on socks & 1 & $3.2 \%$ & 7 & $22.6 \%$ & \\
\hline & Foot callus & 8 & $11.9 \%$ & 1 & $1.5 \%$ & \\
\hline & $\begin{array}{l}\text { Numbness, tingling, pins and needles or itching } \\
\text { sensation in feet }\end{array}$ & 40 & $9.9 \%$ & 29 & $7.2 \%$ & \\
\hline & Tightness, heaviness, pain, or cramps in feet or legs & 35 & $11.7 \%$ & 17 & $5.7 \%$ & \\
\hline
\end{tabular}

*DMD, diabetes mellitus duration

Tightness, heaviness, pain, or cramps of the feet or legs are other major problems among diabetic patients, and approximately $36.5 \%$ of current participants $(21 \%$ female; $16 \%$ male) were affected by this. Moreover, among participants with DM duration >5 years, foot complications were more common among female patients. Conversely, among patients with DM duration>20 years, blood or discharge on the socks were more likely to occur among male participants $(22.6 \%)$. 
Foot ulcers were present in $12 \%$ of female and $9.6 \%$ of male diabetic patients (Table2). Age and residence did not have any significant effect on the development of foot ulcers. In contrast, DM duration was significantly associated with a history of foot ulcers $(p<0.01$; Table 2$)$.

The prevalence of factors in diabetic patients with and without foot ulcers is reported in Table3. The type of current DM treatment varied significantly between those with and without foot ulcers $(p<0.01)$. Diabetic patients who used insulin or a combination of insulin and oral hypoglycemic drugs (OHD) had a higher risk of developing foot ulcers than patients using OHD alone or not taking any drug. There was also a significant association between foot ulcers and a sore or cut on the foot or leg healing over a minimum of 2 weeks $(p<0.01)$. Using the chi-square test of significance, the following variables were significantly associated with foot ulcers: amputation of a toe, foot, or leg; decreased foot sensation to pain and touch; concomitant foot sore or blister; presence of blood or discharge on the socks; foot callus; numbness, tingling, pins and needles, or itching sensation of the feet; tightness, heaviness, pain, or cramps of the feet or legs; and "cannot reach and see the plantar aspect of their feet."

In addition, participants applying moisturizing cream to their feet were more likely to develop foot ulcers than those who did not. The likely hood of developing foot ulcers was also higher among participants who soaked their feet; used medicated products for warts, corns, or calluses; applied moisturizing creams or lotions between the toes; or walked bare foot (Table3).

Table3:-Selected characteristics among diabetic patients with and without foot ulcers.

\begin{tabular}{|c|c|c|c|c|c|c|}
\hline \multirow[t]{3}{*}{ Variables } & \multirow[t]{3}{*}{ Categories } & \multicolumn{4}{|c|}{ Diabetic patients } & \multirow[t]{3}{*}{$p$-value } \\
\hline & & \multicolumn{2}{|c|}{ Without foot ulcer } & \multicolumn{2}{|c|}{ With foot ulcer } & \\
\hline & & $\mathrm{N}$ & $\%$ & $\mathrm{~N}$ & $\%$ & \\
\hline \multirow[t]{4}{*}{ Current DM treatment } & OHD* and insulin & 89 & $81.7 \%$ & 20 & $18.3 \%$ & \multirow[t]{4}{*}{$<0.001$} \\
\hline & Oral hyperglycemic drugs & 427 & $94.3 \%$ & 26 & $5.7 \%$ & \\
\hline & Insulin & 167 & $81.5 \%$ & 38 & $18.5 \%$ & \\
\hline & $\begin{array}{l}\text { Diet and physical activity } \\
\text { (without drugs) }\end{array}$ & 47 & $94.0 \%$ & 3 & $6.0 \%$ & \\
\hline \multirow{2}{*}{$\begin{array}{l}\text { Sore or cut on foot or leg healing } \\
\text { over a minimum of } 2 \text { weeks }\end{array}$} & No & 695 & $96.9 \%$ & 22 & $3.1 \%$ & \multirow[t]{2}{*}{$<0.001$} \\
\hline & Yes & 35 & $35.0 \%$ & 65 & $65.0 \%$ & \\
\hline \multirow[t]{2}{*}{ Amputation of a toe, foot, or leg } & No & 724 & $91.0 \%$ & 72 & $9.0 \%$ & \multirow[t]{2}{*}{$<0.001$} \\
\hline & Yes & 6 & $28.6 \%$ & 15 & $71.4 \%$ & \\
\hline \multirow{2}{*}{$\begin{array}{l}\text { Decreased sensation to pain and } \\
\text { touch in the feet }\end{array}$} & No & 588 & $95.0 \%$ & 31 & $5.0 \%$ & \multirow[t]{2}{*}{$<0.001$} \\
\hline & Yes & 142 & $71.7 \%$ & 56 & $28.3 \%$ & \\
\hline \multirow{2}{*}{ Current foot ulcer, sore, or blister } & No & 679 & $93.5 \%$ & 47 & $6.5 \%$ & \multirow[t]{2}{*}{$<0.001$} \\
\hline & Yes & 51 & $56.0 \%$ & 40 & $44.0 \%$ & \\
\hline \multirow[t]{2}{*}{ Blood or discharge on socks } & No & 719 & $91.5 \%$ & 67 & $8.5 \%$ & \multirow[t]{2}{*}{$<0.001$} \\
\hline & Yes & 11 & $35.5 \%$ & 20 & $64.5 \%$ & \\
\hline \multirow[t]{2}{*}{ Foot callus } & No & 678 & $90.4 \%$ & 72 & $9.6 \%$ & \multirow[t]{2}{*}{0.001} \\
\hline & Yes & 52 & $77.6 \%$ & 15 & $22.4 \%$ & \\
\hline \multirow{2}{*}{$\begin{array}{l}\text { Numbness, tingling, pins and } \\
\text { needles or itching sensation in feet }\end{array}$} & No & 389 & $94.2 \%$ & 24 & $5.8 \%$ & \multirow[t]{2}{*}{$<0.001$} \\
\hline & Yes & 341 & $84.4 \%$ & 63 & $15.6 \%$ & \\
\hline \multirow{2}{*}{$\begin{array}{l}\text { Tightness, heaviness, pain, or } \\
\text { cramps in the feet or legs }\end{array}$} & No & 492 & $94.8 \%$ & 27 & $5.2 \%$ & \multirow[t]{2}{*}{$<0.001$} \\
\hline & Yes & 238 & $79.9 \%$ & 60 & $20.1 \%$ & \\
\hline \multirow{2}{*}{$\begin{array}{l}\text { Cannot reach and see the plantar } \\
\text { aspect of their feet }\end{array}$} & No & 115 & $84.6 \%$ & 21 & $15.4 \%$ & \multirow[t]{2}{*}{0.047} \\
\hline & Yes & 615 & $90.3 \%$ & 66 & $9.7 \%$ & \\
\hline \multirow[t]{2}{*}{ Foot examination } & No & 290 & $90.9 \%$ & 29 & $9.1 \%$ & \multirow[t]{2}{*}{0.248} \\
\hline & Yes & 440 & $88.4 \%$ & 58 & $11.6 \%$ & \\
\hline \multirow[t]{4}{*}{ Frequency of foot examination } & Everyday & 144 & $89.4 \%$ & 17 & $10.6 \%$ & 0.879 \\
\hline & Three to six times per week & 57 & $86.4 \%$ & 9 & $13.6 \%$ & \\
\hline & Two times per week or less & 58 & $90.6 \%$ & 6 & $9.4 \%$ & \\
\hline & $\begin{array}{l}\text { When I have problems with my } \\
\text { foot }\end{array}$ & 180 & $89.1 \%$ & 22 & $10.9 \%$ & \\
\hline Washes feet daily & No & 30 & $83.3 \%$ & 6 & $16.7 \%$ & 0.231 \\
\hline & Yes & 700 & $89.6 \%$ & 81 & $10.4 \%$ & \\
\hline
\end{tabular}


Table3- (continued). Selected characteristics among diabetic patients with and without foot ulcers.

\begin{tabular}{|c|c|c|c|c|c|c|}
\hline \multirow[t]{3}{*}{ Variables } & \multirow[t]{3}{*}{ Categories } & \multicolumn{4}{|c|}{ Diabetic patients } & \multirow{3}{*}{$\begin{array}{l}\text { Significan } \\
\text { ce }\end{array}$} \\
\hline & & \multicolumn{2}{|c|}{ Without foot ulcer } & \multicolumn{2}{|c|}{ With foot ulcer } & \\
\hline & & $\mathrm{N}$ & $\%$ & $\mathrm{~N}$ & $\%$ & \\
\hline \multirow{2}{*}{$\begin{array}{l}\text { Dries area between the toes } \\
\text { well }\end{array}$} & No & 370 & $90.2 \%$ & 40 & $9.8 \%$ & \multirow[t]{2}{*}{0.406} \\
\hline & Yes & 360 & $88.5 \%$ & 47 & $11.5 \%$ & \\
\hline \multirow{2}{*}{$\begin{array}{l}\text { Uses moisturizing cream on } \\
\text { feet }\end{array}$} & No & 374 & $92.8 \%$ & 29 & $7.2 \%$ & \multirow[t]{2}{*}{0.002} \\
\hline & Yes & 356 & $86.0 \%$ & 58 & $14.0 \%$ & \\
\hline \multirow[b]{2}{*}{ Soaks the feet } & No & 458 & $91.4 \%$ & 43 & $8.6 \%$ & \multirow[t]{2}{*}{0.016} \\
\hline & Yes & 272 & $86.1 \%$ & 44 & $13.9 \%$ & \\
\hline \multirow{2}{*}{ 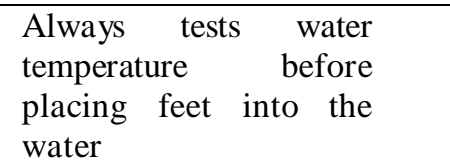 } & No & 344 & $88.9 \%$ & 43 & $11.1 \%$ & \multirow[t]{2}{*}{0.684} \\
\hline & Yes & 386 & $89.8 \%$ & 44 & $10.2 \%$ & \\
\hline \multirow{2}{*}{$\begin{array}{l}\text { Use medicated products for } \\
\text { warts, corns or calluses }\end{array}$} & No & 709 & $90.2 \%$ & 77 & $9.8 \%$ & \multirow[t]{2}{*}{$<0.001$} \\
\hline & Yes & 21 & $67.7 \%$ & 10 & $32.3 \%$ & \\
\hline \multirow{2}{*}{$\begin{array}{l}\text { Applies moisturizing creams } \\
\text { or lotions between the toes }\end{array}$} & No & 502 & $92.1 \%$ & 43 & $7.9 \%$ & \multirow[t]{2}{*}{$<0.001$} \\
\hline & Yes & 228 & $83.8 \%$ & 44 & $16.2 \%$ & \\
\hline \multirow[t]{2}{*}{ Walks barefoot } & No & 285 & $86.1 \%$ & 46 & $13.9 \%$ & \multirow[t]{2}{*}{0.013} \\
\hline & Yes & 445 & $91.6 \%$ & 41 & $8.4 \%$ & \\
\hline \multirow[t]{2}{*}{ Wears shoes without socks } & No & 339 & $89.0 \%$ & 42 & $11.0 \%$ & \multirow[t]{2}{*}{0.745} \\
\hline & Yes & 391 & $89.7 \%$ & 45 & $10.3 \%$ & \\
\hline \multirow{2}{*}{$\begin{array}{l}\text { Always inspects shoes } \\
\text { for foreign objects or } \\
\text { torn linings }\end{array}$} & No & 310 & $87.1 \%$ & 46 & $12.9 \%$ & \multirow[t]{2}{*}{0.064} \\
\hline & Yes & 420 & $91.1 \%$ & 41 & $8.9 \%$ & \\
\hline \multirow{2}{*}{$\begin{array}{l}\text { Uses hot water bottle or } \\
\text { heating pad on feet }\end{array}$} & No & 661 & $89.7 \%$ & 76 & $10.3 \%$ & \multirow[t]{2}{*}{0.344} \\
\hline & Yes & 69 & $86.3 \%$ & 11 & $13.8 \%$ & \\
\hline \multirow[b]{2}{*}{ Sits with legs crossed } & No & 277 & $86.8 \%$ & 42 & $13.2 \%$ & 0.062 \\
\hline & Yes & 453 & $91.0 \%$ & 45 & $9.0 \%$ & \\
\hline & No & 663 & $89.0 \%$ & 82 & $11.0 \%$ & 0.286 \\
\hline Smoker & Yes & 67 & $93.1 \%$ & 5 & $6.9 \%$ & \\
\hline Ever attended a class on & No & 601 & $90.5 \%$ & 63 & $9.5 \%$ & 0.025 \\
\hline how to care for feet & Yes & 129 & $84.3 \%$ & 24 & $15.7 \%$ & \\
\hline If the answer is yes, & No & 223 & $91.8 \%$ & 20 & $8.2 \%$ & 0.157 \\
\hline $\begin{array}{l}\text { applied to activities of } \\
\text { daily living }\end{array}$ & Yes & 116 & $87.2 \%$ & 17 & $12.8 \%$ & \\
\hline Ever read foot care handouts & No & 413 & $87.7 \%$ & 58 & $12.3 \%$ & 0.072 \\
\hline & Yes & 317 & $91.6 \%$ & 29 & $8.4 \%$ & \\
\hline Ever read "proper" foot care & No & 498 & $88.0 \%$ & 68 & $12.0 \%$ & 0.053 \\
\hline handouts & Yes & 232 & $92.4 \%$ & 19 & $7.6 \%$ & \\
\hline Hand out of foot care found & No & 78 & $88.6 \%$ & 10 & $11.4 \%$ & 0.818 \\
\hline useful & Yes & 652 & $89.4 \%$ & 77 & $10.6 \%$ & \\
\hline
\end{tabular}

*OHD, oral hypoglycemic drugs

Factors influencing diabetic foot ulcer:-

The odd ratios (ORs) of factors affecting diabetic foot ulcers are presented in Table4. If all other factors were constant, factors increasing the odds of diabetic foot ulcer included no educational degree (OR, 2.5); use of insulin (OR, 3.6); use of moisturizing cream on the feet $(\mathrm{OR}, 2.1)$; soaking feet $(\mathrm{OR}, 1.7)$; use of medicated products for warts, corns, or calluses $(\mathrm{OR}, 4.4)$; and use of moisturizing creams or lotions between the toes (OR,2.3). Conversely, walking bare foot resulted in a decreased chance (OR, 0.6) of developing foot ulcers.

With regards to adjusted odds ratios (AOR), the risk of developing foot ulcers was increased by the following: a sore or cut on the foot or leg healing over a minimum of 2 weeks (AOR, 32.8); amputation of a toe, 
foot, or leg (AOR, 7); sore or blister on the feet (AOR, 3.5);the presence of blood or discharge on the socks (AOR, 4.5); and tightness, heaviness, pain, or cramps involving the feet or legs (OR, 2.4).

Table4:- Factors associated with diabetic foot ulcer among diabetic patients.

\begin{tabular}{|c|c|c|c|c|c|}
\hline \multirow[t]{2}{*}{ Factor } & \multirow[t]{2}{*}{ Category } & \multicolumn{2}{|c|}{$\begin{array}{l}\text { Have you ever } \\
\text { had a foot ulcer? }\end{array}$} & \multirow[t]{2}{*}{$\mathrm{COR}(95 \% \mathrm{CI})$} & \multirow[t]{2}{*}{$\mathrm{AOR}(95 \% \mathrm{CI})$} \\
\hline & & Yes & No & & \\
\hline \multirow[t]{6}{*}{ Educational status } & Illiterate & 22 & 163 & 1 & \\
\hline & No formal education & 14 & 41 & $2.5 *(1.2,5.4)$ & \\
\hline & Primary school degree & 13 & 80 & $1.2(0.6,2.5)$ & \\
\hline & Secondary school degree & 10 & 73 & $1.0(0.5,2.3)$ & \\
\hline & High school degree & 12 & 185 & $0.5(0.2,1.0)$ & \\
\hline & University degree & 16 & 188 & $0.6(0.3,1.2)$ & \\
\hline \multirow[t]{3}{*}{ Type of diabetes } & Type2 & 15 & 181 & $0.6(0.3,1.0)$ & \\
\hline & Type1 & 10 & 118 & $0.6(0.3,1.2)$ & \\
\hline & Unknown to patient & 62 & 431 & 1 & \\
\hline \multirow{6}{*}{ Duration of diabetes (years) } & $<1$ & 6 & 69 & 1 & \\
\hline & $1-5$ & 10 & 206 & $0.6(0.2,1.6)$ & \\
\hline & $6-10$ & 17 & 180 & $1.1(0.4,2.9)$ & \\
\hline & $11-15$ & 14 & 125 & $1.3(0.5,3.5)$ & \\
\hline & $16-20$ & 15 & 70 & $2.5(0.9,6.7)$ & \\
\hline & $>20$ & 25 & 80 & $3.6 *(1.4,9.3)$ & \\
\hline \multirow[t]{4}{*}{ Current DM treatment } & $\mathrm{OHD}$ and insulin & 20 & 89 & $3.5(1.0,12.5)$ & \\
\hline & $\mathrm{OHD}$ & 26 & 427 & $0.95(0.3,3.3)$ & \\
\hline & Insulin & 38 & 167 & $3.6 *(1.1,121)$ & \\
\hline & $\begin{array}{l}\text { Diet and physical activity } \\
\text { (with out drugs) }\end{array}$ & 3 & 47 & 1 & \\
\hline \multirow{2}{*}{$\begin{array}{l}\text { Sore/cut on foot/leg healing } \\
\text { over a minimum of } 2 \text { weeks }\end{array}$} & No & 22 & 695 & 1 & 1 \\
\hline & Yes & 65 & 35 & $\begin{array}{l}58.7 * *(32.5,105 . \\
9)\end{array}$ & $\begin{array}{l}32.8^{* *}(16.8,63 . \\
8)\end{array}$ \\
\hline \multirow{2}{*}{$\begin{array}{l}\text { Amputation of a toe, foot, or } \\
\text { leg }\end{array}$} & No & 72 & 724 & 1 & 1 \\
\hline & Yes & 15 & 6 & $25.1 * *(9.5,66.8)$ & $7.0 *(1.1,46.2)$ \\
\hline \multirow{2}{*}{$\begin{array}{l}\text { Decreased foot sensation } \\
\text { to pain and touch }\end{array}$} & No & 31 & 588 & 1 & 1 \\
\hline & Yes & 56 & 142 & $7.5 * *(4.7,12.0)$ & $1.9(0.9,4.0)$ \\
\hline \multirow{2}{*}{$\begin{array}{ll}\text { Concomitant footsore } & \text { or } \\
\text { blister }\end{array}$} & No & 47 & 679 & 1 & 1 \\
\hline & Yes & 40 & 51 & $11.3^{* *}(6.8,18.8)$ & $3.5 * *(1.6,7.7)$ \\
\hline \multirow[t]{2}{*}{ Blood or discharge on socks } & No & 67 & 719 & 1 & 1 \\
\hline & Yes & 20 & 11 & $19.5^{* *}(9.0,42.4)$ & $4.5 *(1.2,16.5)$ \\
\hline \multirow[t]{2}{*}{ Foot callus } & No & 72 & 678 & 1 & 1 \\
\hline & Yes & 15 & 52 & $2.7 * *(1.5,5.1)$ & $1.6(0.6,4.4)$ \\
\hline \multirow{2}{*}{$\begin{array}{l}\text { Numbness, tingling, pins and } \\
\text { needles or itching sensation } \\
\text { in feet }\end{array}$} & No & 24 & 389 & 1 & 1 \\
\hline & Yes & 63 & 341 & $3.0 * *(1.8,4.9)$ & $1.4(0.7,3.0)$ \\
\hline
\end{tabular}

Table4:- (continued). Factors associated with diabetic foot ulcer among diabetic patients.

\begin{tabular}{|l|l|l|c|l|}
\hline Factor & Category & $\begin{array}{l}\text { Have you ever } \\
\text { had a foot ulcer? }\end{array}$ & COR(95\%CI) & AOR(95\%CI) \\
\cline { 2 - 3 } & Yes No & \\
\hline
\end{tabular}




\begin{tabular}{|c|c|c|c|c|c|}
\hline \multirow{2}{*}{$\begin{array}{l}\text { Tightness, heaviness, } \\
\text { pain, or cramps in feet } \\
\text { or legs }\end{array}$} & No & 27 & 492 & 1 & 1 \\
\hline & Yes & 60 & 238 & $4.6 * *(2.8,7.4)$ & $2.4 *(1.1,5.0)$ \\
\hline \multirow{2}{*}{$\begin{array}{l}\text { Uses moisturizing cream on } \\
\text { feet }\end{array}$} & No & 29 & 374 & 1 & \\
\hline & Yes & 58 & 356 & $2.1 * *(1.3,3.4)$ & \\
\hline \multirow[t]{2}{*}{ Soaks feet } & No & 43 & 458 & 1 & \\
\hline & Yes & 44 & 272 & $1.7 *(1.1,2.7)$ & \\
\hline \multirow{2}{*}{$\begin{array}{l}\text { Uses medicated products for } \\
\text { warts, cornsorcalluses }\end{array}$} & No & 77 & 709 & 1 & \\
\hline & Yes & 10 & 21 & $4.4 * *(2 \cdot 0,9.7)$ & \\
\hline \multirow{2}{*}{$\begin{array}{l}\text { Applies } \quad \text { moisturizing } \\
\text { creams/lotions between the } \\
\text { toes }\end{array}$} & No & 43 & 502 & 1 & \\
\hline & Yes & 44 & 228 & $2.3 * *(1.4,3.5)$ & \\
\hline \multirow[t]{2}{*}{ Walks barefoot } & No & 46 & 285 & 1 & \\
\hline & Yes & 41 & 445 & $0.6 *(0.4,0.9)$ & \\
\hline
\end{tabular}

*Oddsratiosignificantat5\%level. **Oddsratiosignificantat1\%level. A value of 1 indicates the reference group.CI, confidence interval; OHD, oral hypoglycemic drugs; AOR, adjusted odds ratio; COR, crude (unadjusted) odds ratio.

\section{Discussion:-}

The development of most DM foot complications can be prevented. Recent research found that, globally, diabetic foot ulcer prevalence was 6.3\%; Australia had the lowest prevalence at $1.5 \%$, while Belgium had the highest prevalence at $16.6 \%$, followed by Canada at $14.8 \%$ and the United States (US)at $13 \%$ (14). The current study found that the prevalence of diabetic foot ulcer in the Qassim Region, Saudi Arabia, was 10.8\%, which is within the global prevalence range. Moreover, we found a high prevalence of peripheral neuropathy, affecting approximately half of diabetic patients $(49.5 \%)$. Of these, $60 \%$ of patients were female and $40 \%$ were male. The prevalence of foot ulcers was higher among female (12.0\%) than among male participants $(9.6 \%)$.

The current study aimed to assess the prevalence of characteristics regarding foot care in diabetic patients in the Qassim Region of Saudi Arabia. It also aimed to assess factors associated with diabetic foot ulcers. The influence of sex on foot ulcers is controversial: a number of studies have demonstrated that male sex is a risk factor, while other studies have shown no effect of sex (15). However, contrary to this study, most previous studies have found that male patients have a higher risk of developing foot ulcers than female patients (16). This difference may be explained by the local culture in the Qassim Region. Here, females remain primarily indoors and do not often engage in extracurricular activities, owing to the lack of female health clubs and public running courses. Another potential explanation may be related to poor eating habits and intake of unhealthy, fast foods.

In this study, we found that age distribution did not have any significant effect on the development of foot ulcers; therefore, foot ulcers can occur at any age. This finding corroborates the results of a UK study (17). However, other studies have reported that age is an important risk factor for foot ulcers $(12,13,18)$.

DM duration was associated with increased foot ulcer prevalence; specifically, participants with DM duration of $>20$ years were 3.6 times more likely to develop foot ulcers than participants with DM duration of 0-5 years. This is consistent with the results of other studies $(18,19)$. This may be because increased DM duration results in added risk for the development of diabetic complications, such as neuropathy and peripheral vascular disease, which contribute to the progression of foot ulcers in DM patients.

We also found that the current type of diabetic treatment was significantly associated with the development of foot ulcers. Specifically, insulin or the combination of insulin and OHD were associated with an increased chance of developing foot ulcers, compared to the use of no drugs. This is consistent with the results of another study, which concluded that insulin use was a significant risk factor for diabetic foot ulcers (18).

The current data also show that participants with no education were 2.5 times more likely to develop diabetic foot ulcers; this is consistent with previous studies reporting that the prevalence of foot lesions was greater among patients with no education than among patient with a degree $(20,21)$. Moreover, participants who used moisturizers were 2.5 times more likely to develop diabetic foot ulcers. This finding was surprising, as medical DM foot guidelines recommend the daily use of moisturizers (22). To the best of our knowledge, there are no reports in the 
literature regarding the association between the use of moisturizing creams on the foot and the development of diabetic foot disease. We postulate that patients using moisturizing creams may have applied the cream inappropriately between the toes, promoting fungal infection and leading to ulceration. Research conducted in Ethiopia reported that patients with dry foot skin were 3.5 times more likely to develop foot ulcers than those with smooth, moist foot skin (23); therefore, this finding remains controversial.

The presence of a foot wound or cuts healing over a minimum of 2 weeks was associated with a 32.8 times increased risk of developing foot ulcers, compared to the risk among patients with no foot wounds. This was the highest risk factor for developing foot ulcers in the current study. The likely explanation is that diabetic patients experience delayed healing process at the cellular level, including disturbed microcirculation, reduced fibroblast proliferation, reduced inflammatory reaction, and altered cytokine-protease profile (24). Furthermore, other factors that impair wound healing, such as age, nutritional status, and hyperglycemia, contribute to the progression from unhealed wound to ulcer.

Participants with tightness, heaviness, pain, or cramps in the feet or legs and patients with numbness, tingling, pins and needles, or itching sensation in the feet had at increased odds of developing diabetic foot ulcers. This finding is consistent with previous studies reporting that $67 \%$ of foot ulcers were neuropathic (25). Moreover, there were increased odds of developing diabetic foot ulcers among patients with a previous amputation of a toe, foot, or leg, as well as among those with a history of foot sore or blisters. A study in the UK showed that a history of ulceration was the highest risk factor for the incidence of foot ulcers (26).In the same study, it was reported that a callus on the foot should be established as a high-risk factor for foot ulcers. The findings of present study support this recommendation.

The current results indicate that smoking was not a statistically significant risk factor for diabetic foot ulcer. These findings corroborate a study conducted in the US (17). Conversely, a further US study reported that diabetic patients who smoke were more likely to undergo a lower extremity amputation than non-smoker diabetic patients; the study also identified a trend towards more proximal amputation among diabetic smokers, although this was not statistically significant (27).

\section{Conclusion:-}

The prevalence of diabetic foot ulcer in the Qassim Region is $10.8 \%$, which is within the global prevalence range. This study found a high prevalence of peripheral neuropathy, affecting almost half of diabetic patients (49.5\%) and predominantly affecting female patients. Female diabetic patients are more likely to develop foot ulcers than male patients. Our data highlight that multiple factors are implicated in the development of DM foot ulcers and, specifically, foot wound or cuts healing over a minimum of 2 weeks and the presence of blood or discharge on the socks were more strongly associated with the development of foot ulcers.

\section{Acknowledgments:-}

We would like to express our deepest appreciation to Nada A Al Dhuwaihi, Sadeem A Alrobiaee, Aljowharah S Alotaibi, Abdullah M Almoziraei, Eisa A Alromaih, and Bassam A Alghidani who helped collect the data. Furthermore, I would also like to acknowledge the crucial role of my colleagues, Fahad AAlsaawi, Razan A Alharbi, Abdullah S Almazied, Abdulrahman M Alrshody, and Meshal M Alrumayh.

\section{References:-}

1. Guariguata L, Whiting D, Hambleton I, Beagley J, Linnenkamp U, Shaw J. Global estimates of diabetes prevalence for 2013 and projections for 2035. Diabetes Res ClinPract 2014;103:137-49.

2. Arab M. The economics of diabetes care in the Middle East. In: Alberti K, Zimmet P, Defronzo R, eds. International Textbook of Diabetes Mellitus. 2nd ed. John Wiley and Sons, Chichester 1997; 1047-1053.

3. World Bank. World Bank Data, WHO parameters, 1999-2000.World bank; 2000.

4. Saudi Arabia. International Diabetes Federation. 2016 [cited 12 March 2016]. Available from: http://www.idf.org/membership/mena/saudi-arabia

5. Alhowaish A. Economic costs of diabetes in Saudi Arabia. J Fam Community Med 2013;20:1.

6. - Diabetes. 1st ed. Belgium: International Working Group on the Diabetic Foot; 2016 [cited 5 March 2016]. Available from: https://www.idf.org/webdata/docs/background_info_AFR.pdf 
7. Fedele D, Comi G, Coscelli C, Cucinotta D, Feldman E, Ghirlanda G, et al. A Multicenter Study on the Prevalence of Diabetic Neuropathy in Italy. Diabetes Care 1997; 20: 836-3.

8. Abdullah M Alwahabi.The diabetic foot in the Arab world. Saudi Med J 2006; 27:147-53.

9. Bantomane A, Mohammedi F, Ayed F, Kadi K, Azzouz A. Diabetic foot lesions, etiologic and prognostic factors. Diabetes Metab 2000; 26:113-7.

10. Nielsen JV.Peripheral neuropathy, hypertension, foot ulcers and amputation among Saudi Arabian patients with type 2 diabetes. Diabetes Res ClinPract 1998; 41:63-9.

11. Halawa M, Karawagh A, Zeidan A, Mahmoud AE, Sakr M, Hegazy A. Prevalence of painful diabetic peripheral neuropathy among patients suffering from diabetes mellitus in Saudi Arabia. Curr Med Res Opin 2010;26:33743.

12. Dixit S, Maiya A, Khetrapal H, Agrawal B, Vidyasagar S, Umakanth S.A questionnaire based survey on awareness of diabetic foot care in Indian population with diabetes: a cross-sectional multicenter study. Indian J Med Sci 2011;65:411-23.

13. Al-Busaidi I. Nzresearch.org.nz. 2014 [cited 3 June 2016]. Available from: http://www.nzresearch.org.nz/records?i\%5Bcreator\%5D=Al-

Busaidi\%2C+Ibrahim+Saleh\&i\%5Bthesis_level\%5D=Undergraduate\&locale=en\&recordset=research

14. Zhang P e. Global Epidemiology of Diabetic Foot Ulceration: A Systematic Review and Meta-Analysis. PubMed - NCBI [Internet]. Ncbi.nlm.nih.gov. 2016 [cited 16 September 2016]. Available from: https://www.ncbi.nlm.nih.gov/pubmed/27585063

15. Dinh T, Veves A. The influence of gender as a risk factor in diabetic foot ulceration. Wounds 2008; 20: 127-31.

16. Peek M. Gender Differences in Diabetes-related Lower Extremity Amputations. ClinOrthopRelRes2010;469:1951-5.

17. Boyko E, Ahroni J, Stensel V. A prospective study of risk factors for diabetic foot ulcer. The Seattle Diabetic Foot Study. Diabetes Care 1999;22:1036-42.

18. Shahi SK, Kumar A, Kumar S, Singh SK, Gupta SK, Singh TB. Prevalence of Diabetic Foot Ulcer and Associated Risk Factors in Diabetic Patients From North India. J Diabetic Foot Complic2012; 4: 83-91.

19. Khan H, Khan Z, Khan I, Jamal ud Din, Rehman S, Khan B. Factors contributing to the development of diabetic foot ulcers and role of health literacy. Rawal Med J 2011; 36: 34-7.

20. de Lima I, Peixoto A, de Oliveira A, Borges J, da Costa J, Pancieri M, et al. Relationship between theeducation level and dermatological lesions in feet of diabetic patients. DiabetolMetabSyndr2015;7: A187.

21. Al-Maskari F, El-Sadig M. Prevalence of risk factors for diabetic foot complications. BMC Fam Pract2007;8: 59.

22. Foot Complications. American Diabetes Association. 2016 [cited 17 September 2016].Available from: http://www.diabetes.org/living-with- diabetes/complications/foot-complications/

23. Deribe B. Prevalence and Factors Influencing Diabetic Foot Ulcer among Diabetic Patients AttendingArbaminch Hospital, South Ethiopia. J Diabetes Metab 2014;05: 10000322.

24. Volmer-Thole M, Lobmann R. Neuropathy and Diabetic Foot Syndrome. Int J Mol Sci. 2016;17:917.

25. Oyibo S, Jude E, Tarawneh I. The effects of ulcer size and site, patient's age, sex and type and duration of diabetes on the outcome of diabetic foot ulcers. Diabetic Med 2001;18:133-8.

26. Murray H, Young M, Hollis S. The association between callus formation, high pressures and neuropathy in diabetic foot ulceration. Diabetic Med 1996; 13:979-82.

27. Anderson J, Boone J, Hansen M. A comparison of diabetic smokers and non-smokers who undergo lower extremity amputation: a retrospective review of 112 patients. Diabet Foot Ankle 2012;3. 